\title{
Fatores associados ao tempo de tela em estudantes do ensino médio de Fortaleza, região Nordeste do Brasil
}

\author{
Factors associated with screen time among high school students \\ in Fortaleza, Northeastern Brazil
}

\author{
Jéssica dos Santos Martins ${ }^{1,4} \bowtie$, Evanice Avelino de Souza ${ }^{2,4}$, Nicolino Trompieri Filho ${ }^{3,4}$ \\ ${ }^{1}$ Curso de Educação Física da Faculdade Terra Nordeste (FATENE). Fortaleza, CE. \\ 2 Programa de Pós Graduação em Ciências Médicas, Faculdade de Medicina, Universidade Federal do Ceará (UFC). Fortaleza, CE. \\ ${ }^{3}$ Programa de Pós-Graduação em Educação Brasileira da Universidade Federal do Ceará (UFC). Fortaleza, CE. \\ ${ }^{4}$ Grupo de Pesquisa em Promoção da Atividade Física e Saúde na Escola (GPAFSE/UFC). Fortaleza, CE.
}

Este estudo teve apoio financeiro do Conselho Nacional de Desenvolvimento Científico e Tecnológico (CNPq).

\section{RESUMO}

Objetivos: Investigar os fatores associados ao tempo em que os escolares despendem assistindo televisão, usando computador ou jogando videogames.

Métodos: Estudo transversal com adolescentes matriculados no ensino médio, selecionados aleatoriamente em quatro escolas da rede Liceu do Ceará, na cidade de Fortaleza, Nordeste do Brasil. O número de estudantes convidados a participar do estudo foi proporcional à amostra representativa da escola, sendo convidados aleatoriamente. Os dados levantados foram autorreportados e incluíram: duração diária do tempo de tela (tempo passado por dia de semana assistindo televisão ou usando videogame / computador), consumo de refrigerantes, doces e salgados, sexo, idade, turno de estudo, status ocupacional, participação nas aulas de Educação Física e prática de atividade física extracurricular. O teste de Qui-quadrado para tendência linear foi utilizado para analisar as associações e o nível de significância foi estipulado em $\mathrm{p} \leq 0,05$.

Resultados: Foram avaliados 964 escolares, sendo 46,4\% na faixa etária entre 17 e 19 anos e 51,5\% do sexo feminino. A prevalência de estudantes que assistiam televisão por mais de três horas diárias foi de $51,1 \%$ e a maior proporção foi observada entre adolescentes mais novos de ambos os sexos, que não trabalhavam, estudavam no turno da tarde e consumiam refrigerantes, doces e salgados frequentemente. O uso excessivo de computador/videogame esteve presente em $21,85 \%$ da amostra, com maior prevalência entre meninos do turno da manhã e que consumiam salgados frequentemente.

Conclusões: Fatores sociodemográficos e comportamentais, incluindo hábitos alimentares, estão associados ao tempo de tela em adolescentes. Contudo, os fatores associados diferiram de acordo com o tipo de aparelho mais utilizado (televisão ou computador/videogame). Estes aspectos devem ser considerados em programas de combate ao tempo sedentário no lazer em jovens.

DESCRITORES: comportamento do adolescente; saúde do adolescente; jogos de vídeo; televisão; preferências alimentares; estudos transversais.

\section{ABSTRACT}

Aims: To investigate the factors associated with the time adolescents spend watching television, using the computer, or playing video games. Methods: Cross-sectional study with adolescents attending high school, randomly selected from four schools of the Liceu do Ceará network in the city of Fortaleza, Northeastern Brazil. The number of students invited to participate in the study was proportional to the representative sample of the school. All the data were self-reported, including: daily screen time (time spent watching television or using the video game or the computer), intake of soft drinks, sweets, and packaged snack foods, sex, age, school shift, occupational status, participation in physical education classes, and practice of extracurricular physical activity. The chi-square test for linear trend was used to assess the associations, and the significance level was set as $\mathrm{p}<0.05$.

Results: A total of 964 students (46.4\% aged 17 to 19 years and $51.5 \%$ female) were assessed. The prevalence of students who watched TV for more than 3 hours a day was $51.1 \%$ and the highest rate was observed among younger adolescents of both sexes, who did not work, went to school in the afternoon, and often consumed soft drinks, sweets, and packaged snack foods. Excessive use of computer / video games was observed in $21.85 \%$ of the sample, with a higher prevalence among boys who attended school in the morning and regularly consumed packaged snack foods.

Conclusions: Sociodemographic and behavioral factors, including eating habits, are associated with screen time among adolescents. However, associated factors differed depending on the most widely type of media used (TV, computer, or video games). These aspects should be considered in programs for the elimination of leisure time sedentary behavior among adolescents.

KEY WORDS: adolescent behavior; adolescent health; video games; television; food preferences; cross-sectional studies. 
Abreviaturas: TV, televisão; CDC, Centers for Disease Control and Prevention.

\section{INTRODUÇÃO}

A inatividade física tem sido apontada como o quarto principal fator de risco para a mortalidade global. Embora exista forte relação entre inatividade física e doenças crônicas não transmissíveis, estudos têm apontado alarmantes prevalências de sedentarismo, tanto em adultos quanto em crianças e adolescentes [1]. Essas estimativas podem ser explicadas, em parte, pela maior disponibilidade e utilização de recursos eletrônicos, como televisão (TV), computador e videogame $[2,3]$.

Uma pesquisa realizada em 2009 pelo Youth Risk Behavior Survey, um programa do Centers for Disease Control and Prevention (CDC) [4], com adolescentes norte-americanos, encontrou alto índice de excessivo tempo de tela (tempo em que um indivíduo utiliza $\mathrm{TV} /$ videogame/computador). Entre os adolescentes pesquisados, 24,9\% usavam computador/videogame e $32,8 \%$ assistiam TV por três ou mais horas diárias. No entanto, essas estimativas são inferiores às encontradas na maioria dos estudos realizados com adolescentes brasileiros [5-8]. Portanto, fica evidente que o comportamento sedentário também está presente em grandes proporções na população jovem brasileira, sendo necessários estudos que busquem apontar os fatores associados a este comportamento.

Contudo, alguns estudos [2,9-15] limitaram-se à análise de um único indicador de tempo de tela, ou seja, levando em conta apenas um tipo de aparelho (TV, computador ou videogame). São necessários estudos para avaliar se os fatores associados podem variar segundo os tipos de aparelhos mais utilizados pelos adolescentes. Tais estudos permitirão o direcionamento de intervenções aos subgrupos de maior risco conforme o indicador. Considerando estes aspectos, o presente estudo teve como objetivo investigar os fatores associados ao tempo de tela excessivo (considerando TV ou computador/ videogame) em uma amostra de estudantes do ensino médio de Fortaleza, na região Nordeste do Brasil.

\section{MÉTODOS}

Este estudo apresentou delineamento transversal e fez parte de um projeto de pesquisa intitulado "Análise de classificação nutricional, hábitos alimentares, práticas de atividade física e comportamento sedentário em escolares do colégio Liceu do Ceará". Neste estudo foram focadas as sedes do Liceu do Ceará, uma rede de ensino público localizada na cidade de Fortaleza, região Nordeste do Brasil e a sétima maior cidade do país [16]. As sedes do Liceu Ceará oferecem ensino médio completo e curso pré-vestibular. Quatro sedes foram estudadas: Liceu Centro, Liceu do Conjunto Ceará, Liceu de Messejana e Liceu do bairro Vila Velha. Estas sedes conseguem agregar estudantes de diferentes bairros e regiões metropolitanas de Fortaleza.

Em 2010 existiam 6.933 alunos matriculados nas quatro sedes do Liceu do Ceará. A estimativa do tamanho amostral necessário para o estudo foi efetuada conforme o número de escolares matriculados em cada sede. Foram considerados os seguintes parâmetros estatísticos: (a) população de 6.933 adolescentes; (b) nível de confiança de 95\%; (c) erro amostral de três pontos percentuais; e (d) prevalência de tempo excessivo de tela de 50\% (a qual obtém variância máxima). No total, a amostra mínima para o estudo foi estimada em 926 adolescentes (Tabela 1).

Tabela 1. Cálculo amostral para recrutamento de estudantes de ensino médio para o estudo em cada sede do Liceu do Ceará, em Fortaleza, estado do Ceará, região Nordeste do Brasil.

\begin{tabular}{lccc}
\hline \multicolumn{1}{c}{ Colégio } & População & $\begin{array}{c}\text { Amostra } \\
\text { calculada }\end{array}$ & $\begin{array}{c}\text { Amostra } \\
\text { estudada }\end{array}$ \\
\hline Liceu Centro & 3700 & 494 & 500 \\
\hline Liceu Messejana & 1318 & 176 & 185 \\
\hline Liceu Conjunto Ceará & 1144 & 153 & 165 \\
Liceu Vila Velha & 771 & 103 & 114 \\
\hline Total & 6.933 & 926 & 964 \\
\hline
\end{tabular}

A pesquisa foi aprovada pelo Comitê de Ética em Pesquisa da Universidade Federal do Ceará sob parecer consubstanciado de número 45/10. Os pais ou responsáveis dos adolescentes assinaram um termo de consentimento livre e esclarecido e os adolescentes concordaram em participar do estudo.

A coleta de dados foi realizada de fevereiro a junho de 2011, quando os adolescentes realizaram preenchimento de questionários em sala de aula, sendo supervisionados por uma equipe de profissionais e acadêmicos de Educação Física previamente treinados. Foram realizadas duas visitas à cada sede do Liceu do Ceará. A primeira para apresentação dos objetivos da pesquisa e entrega do termo de consentimento aos escolares. A segunda visita foi utilizada para a coleta de dados. Estudantes dos três anos do ensino médio foram convidados, de todas as turmas das escolas. Os estudantes eram recrutados aleatoriamente pelo professor que estava em sala de aula. Solicitávamos cinco ou oito escolares por sala, independentemente do 
sexo. Caso o apontado tivesse faltado ou se recusasse a participar, era chamado outro.

O tempo de tela foi obtido pelo tempo em que o adolescente assistia TV e jogava videogame/ computador em um dia de semana. Utilizaram-se as seguintes questões: 1) Quanto tempo, por dia, você assiste TV? 2) Quanto tempo, por dia, você passa jogando/usando videogame/computador? Para as duas questões consideraram-se apenas os dias de semana e não os de fim de semana. Os adolescentes que referiram passar três horas ou mais por dia em tempo de tela foram considerados com tempo excessivo, conforme as recomendações do CDC [17].

Os dados sobre hábitos alimentares foram obtidos por meio de três questões relacionadas aos seguintes alimentos: refrigerantes, doces e salgados fritos (como coxinhas, pasteis, rissoles e demais tipos de salgados que fossem fritos). Em cada questão, o estudante respondia sobre a frequência de consumo do alimento em uma semana habitual. Essas questões foram baseadas em instrumentos previamente validados, aplicados com adolescentes brasileiros [2,3].

Também foram anotados, sexo, idade, turno de aula (manhã, tarde ou noite), ocupação profissional (trabalha ou não trabalha), prática de atividade física extracurricular e participação nas aulas de Educação Física escolar (participa ou não participa). Estas informações foram autorreportadas, de acordo com instrumentos previamente validados e aplicados a escolares brasileiros [18].

A estatística descritiva foi utilizada para apresentação dos dados através da frequência absoluta e relativa. $\mathrm{O}$ teste de qui-quadrado foi utilizado para identificar a associação entre o tempo excessivo de tela e as variáveis categóricas. O nível de significância foi estabelecido em $5 \%(\mathrm{p}<0,05)$ e as análises foram feitas através do software estatístico IBM-SPSS versão 21.0.

\section{RESULTADOS}

Foram estudados 964 adolescentes, sendo 497 (51,5\%) do sexo feminino. A maior parte da amostra foi de estudantes com idades entre 17 e 19 anos, do turno da tarde e que não trabalhavam. A maior proporção de estudantes praticava atividade física extracurricular e participava das aulas de Educação Física escolar (Tabela 2).

Tabela 2. Características demográficas e comportamentais de 964 estudantes de ensino médio do Liceu do Ceará, em Fortaleza, participantes do estudo

\begin{tabular}{|c|c|c|c|c|c|c|c|}
\hline \multirow{2}{*}{ Variáveis } & \multicolumn{2}{|c|}{ Total } & \multicolumn{2}{|c|}{ Rapazes } & \multicolumn{2}{|c|}{ Moças } & \multirow{2}{*}{$\mathbf{p}^{*}$} \\
\hline & n & $\%$ & $\mathbf{n}$ & $\%$ & n & $\%$ & \\
\hline \multicolumn{8}{|l|}{ Faixa etária } \\
\hline 14-16 anos & 417 & 43,3 & 188 & 45,1 & 229 & 54,9 & \multirow{3}{*}{0,01} \\
\hline 17-19 anos & 447 & 46,4 & 240 & 53,7 & 207 & 46,3 & \\
\hline$\geq 20$ anos & 100 & 10,4 & 39 & 39,0 & 61 & 61,0 & \\
\hline \multicolumn{8}{|l|}{ Trabalha } \\
\hline Não & 648 & 67,2 & 304 & 46,9 & 344 & 53,1 & \multirow{2}{*}{0,09} \\
\hline Sim & 316 & 32,8 & 163 & 51,6 & 153 & 48,4 & \\
\hline \multicolumn{8}{|l|}{ Turno de estudo } \\
\hline Manhã & 330 & 34,2 & 161 & 48,8 & 169 & 51,2 & \multirow{3}{*}{0,45} \\
\hline Tarde & 334 & 34,6 & 169 & 50,6 & 165 & 49,4 & \\
\hline Noite & 300 & 31,1 & 137 & 45,7 & 163 & 54,3 & \\
\hline \multicolumn{8}{|c|}{ Participa das aulas de Educação Física } \\
\hline Sim & 275 & 71,3 & 162 & 58,9 & 113 & 41,1 & \multirow{2}{*}{0,01} \\
\hline Não & 684 & 28,7 & 303 & 44,3 & 381 & 55,7 & \\
\hline \multicolumn{8}{|c|}{ Pratica atividade física extracurricular } \\
\hline Sim & 263 & 72,7 & 176 & 66,9 & 87 & 33,1 & \multirow{2}{*}{0,01} \\
\hline Não & 701 & 27,3 & 291 & 41,5 & 410 & 58,5 & \\
\hline \multicolumn{8}{|c|}{ Assiste TV em dias de semana } \\
\hline$<3$ horas dia & 471 & 48,9 & 233 & 49,5 & 238 & 50,5 & \multirow{2}{*}{0,53} \\
\hline$\geq 3$ horas dia & 493 & 51,1 & 234 & 47,5 & 259 & 52,5 & \\
\hline \multicolumn{8}{|c|}{ Usa VG/PC em dias de semana } \\
\hline$<3$ horas dia & 754 & 78,2 & 339 & 45,0 & 415 & 55,0 & \multirow{2}{*}{0,01} \\
\hline$\geq 3$ horas dia & 210 & 21,8 & 128 & 61,0 & 82 & 39,0 & \\
\hline
\end{tabular}

TV, televisão; VG, videogame; PC, computador; * Teste do qui-quadrado. 
A prevalência de estudantes que assistiam TV por mais de três horas diárias foi de $51,1 \%$, e a prevalência de estudantes que usavam computador/videogame por mais de três horas diárias foi de $21,8 \%$. O tempo excessivo de TV foi observado na maioria dos adolescentes e de modo semelhante entre moças e rapazes. Em contrapartida, o uso excessivo de computador/videogame esteve presente em 21,8 \% da amostra, com maior prevalência entre meninos (Tabela 2).
Estudantes mais novos, que não trabalhavam, que estudavam a tarde e que consumiam refrigerantes, doces e salgados frequentemente, foram os que tiveram maior proporção de tempo de TV de três ou mais horas diárias. Adolescentes mais novos, do turno da manhã e que consumiam salgados frequentemente, tiveram maiores prevalências de tempo usando computador/videogame por três ou mais horas diárias (Tabela 3).

Tabela 3. Frequência absoluta e relativa do tempo excessivo de televisão e videogame/computador de acordo com as variáveis independentes, em 964 estudantes do ensino médio do Liceu do Ceará, em Fortaleza, Ceará, região Nordeste do Brasil.

\begin{tabular}{|c|c|c|c|c|c|c|c|c|c|c|}
\hline \multirow{3}{*}{ Variáveis } & \multicolumn{5}{|c|}{ Televisão } & \multicolumn{5}{|c|}{ Videogame/computador } \\
\hline & \multicolumn{2}{|c|}{$<3$ horas/dia } & \multicolumn{2}{|c|}{$\geq 3$ horas/dia } & \multirow{2}{*}{$\mathbf{p}^{*}$} & \multicolumn{2}{|c|}{$<3$ horas/dia } & \multicolumn{2}{|c|}{$\geq 3$ horas/dia } & \multirow{2}{*}{$\mathbf{p}^{*}$} \\
\hline & $\mathbf{n}$ & $\%$ & n & $\%$ & & n & $\%$ & n & $\%$ & \\
\hline \multicolumn{11}{|l|}{ Faixa etária } \\
\hline 14-16 anos & 191 & 45,8 & 226 & 54,2 & \multirow{3}{*}{0,05} & 332 & 79,6 & 85 & 20,4 & \multirow{3}{*}{0,02} \\
\hline 17-19 anos & 221 & 49,4 & 226 & 50,6 & & 332 & 74,3 & 115 & 25,7 & \\
\hline$\geq 20$ anos & 59 & 59,0 & 41 & 41,0 & & 90 & 90,9 & 10 & 10,0 & \\
\hline \multicolumn{11}{|l|}{ Trabalha } \\
\hline Sim & 200 & 63,3 & 116 & 37,6 & \multirow{2}{*}{0,01} & 249 & 78,8 & 67 & 21,2 & \multirow{2}{*}{0,76} \\
\hline Não & 271 & 41,8 & 377 & 58,2 & & 505 & 77,9 & 143 & 22,1 & \\
\hline \multicolumn{11}{|l|}{ Turno de estudo } \\
\hline Manhã & 167 & 50,6 & 163 & 49,4 & \multirow{3}{*}{0,01} & 241 & 73,0 & 89 & 27,0 & \multirow{3}{*}{0,01} \\
\hline Tarde & 137 & 41,0 & 197 & 59,0 & & 258 & 77,2 & 76 & 22,8 & \\
\hline Noite & 167 & 55,7 & 133 & 44,3 & & 255 & 85,0 & 45 & 15,0 & \\
\hline \multicolumn{11}{|c|}{$\begin{array}{l}\text { Participação nas aulas de Educação } \\
\text { Física na escola }(n=959)\end{array}$} \\
\hline $\operatorname{Sim}$ & 120 & 43,6 & 155 & 56,4 & \multirow{2}{*}{0,04} & 219 & 79,6 & 56 & 20,4 & \multirow{2}{*}{0,49} \\
\hline Não & 347 & 50,7 & 337 & 49,3 & & 531 & 77,6 & 153 & 22,4 & \\
\hline \multicolumn{11}{|c|}{$\begin{array}{l}\text { Prática de atividade física } \\
\text { extracurricular }\end{array}$} \\
\hline Sim & 129 & 49,0 & 134 & 51,0 & \multirow{2}{*}{0,94} & 195 & 74,1 & 68 & 25,9 & \multirow{2}{*}{0,06} \\
\hline Não & 342 & 48,8 & 359 & 51,2 & & 559 & 79,7 & 142 & 20,3 & \\
\hline \multicolumn{11}{|l|}{ Consumo de refrigerante } \\
\hline Não consome & 85 & 60,3 & 56 & 39,7 & \multirow{4}{*}{0,02} & 114 & 80,9 & 27 & 19,1 & \\
\hline 1 a $3 \times$ / semana & 201 & 48,7 & 212 & 51,3 & & 332 & 80,4 & 81 & 19,6 & 0 \\
\hline 4 a $6 \times$ / semana & 87 & 43,9 & 111 & 56,1 & & 146 & 73,7 & 52 & 26,3 & 0,21 \\
\hline Diariamente & 98 & 46,2 & 114 & 53,8 & & 162 & 76,4 & 50 & 23,6 & \\
\hline Consumo de doces & & & & & & & & & & \\
\hline Não consome & 83 & 62,9 & 49 & 37,1 & & 104 & 78,8 & 28 & 21,2 & \\
\hline 1 a $3 \times$ / semana & 237 & 53,4 & 207 & 46,6 & 001 & 356 & 80,2 & 88 & 19,8 & 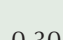 \\
\hline 4 a $6 \times$ / semana & 61 & 43,9 & 78 & 56,1 & 0,01 & 101 & 72,7 & 38 & 27,3 & 0,30 \\
\hline Diariamente & 90 & 36,1 & 159 & 63,9 & & 193 & 77,5 & 56 & 22,5 & \\
\hline Consumo de salgadost & & & & & & & & & & \\
\hline Não consome & 99 & 59,6 & 67 & 40,4 & & 143 & 86,1 & 23 & 13,9 & \\
\hline 1 a $3 \times$ / semana & 212 & 48,2 & 228 & 51,8 & 0 & 356 & 80,9 & 84 & 19,1 & , \\
\hline 4 a $6 \times$ / semana & 80 & 49,7 & 81 & 50,3 & 0,07 & 110 & 68,3 & 51 & 31,7 & 0,0 \\
\hline Diariamente & 80 & 40,6 & 117 & 59,4 & & 145 & 73,6 & 52 & 26,4 & \\
\hline
\end{tabular}

* Teste do qui-quadrado; + Coxinha, pastel, rissole e outros salgados fritos. 


\section{DISCUSSÃO}

Este estudo investigou fatores associados ao tempo de tela excessivo em adolescentes do ensino médio de Fortaleza, Ceará, na região Nordeste do Brasil. De modo geral, fatores sociodemográficos (idade, sexo, situação de trabalho) e comportamentais (atividade física, hábitos alimentares), estiveram associados ao tempo de tela entre os adolescentes.

É comum o adolescente passar muito tempo assistindo TV, utilizando computador ou jogando com videogame. No intervalo em que o jovem não está na escola, os pais geralmente permitem que ele fique em frente às telas, o que pode ser justificado pela falta de locais de lazer seguros e atrativos, falta de apoio e orientação dos adultos ou, ainda, ausência de conhecimento quanto os benefícios da atividade física. Neste estudo, o tempo de tela total não foi diferente entre os sexos, mas os meninos, independente da idade, passavam mais tempo usando computador ou videogame quando comparados às meninas, que passavam mais tempo assistindo TV. Em relação a este dado, um estudo longitudinal realizado com escolares da Alemanha verificou um declínio no tempo de TV durante a semana e aos finais de semana, principalmente entre as meninas. Aquele mesmo estudo verificou um aumento no tempo do uso de computador, principalmente devido à utilização da Internet, e-mails, trabalhos de casa e conversas online, apontando a necessidade de estratégias para reduzir o tempo de tela [19].

O presente estudo identificou uma associação significativa entre adolescentes que trabalham e que estudam a noite com passar menor tempo em frente à $\mathrm{TV}$, sugerindo que o comportamento de assistir TV em excesso na adolescência passa a apresentar declínio quando o adolescente inicia a carreira profissional concomitantemente ao período letivo, ocupando o turno não dedicado à escola. Verifica-se nessa etapa uma maior autonomia do adolescente, que passa a vivenciar longos períodos do dia fora de casa e consequentemente menos tempo despendido dentro de casa em frente às telas [10].

Este estudo mostrou também uma associação entre consumo de alimentos não saudáveis e tempo excessivo assistindo TV, o que também foi encontrado em um estudo com crianças e adolescentes no Canadá, que identificou uma associação significativa do consumo de alimentos não saudáveis (doces, refrigerantes e batatas fritas) com maior tempo em frente à TV [14]. Outro estudo, realizado em cidades europeias, também identificou uma associação entre o tempo excessivo de TV e a ingestão de alimentos ricos em açúcar e gorduras, podendo ocasionar excesso de peso [15].

O tempo em frente à TV associado ao consumo de alimentos não saudáveis pode ser explicado por diversos fatores, como comerciais, sensação de saciedade [9] e hábitos dos pais em realizar as refeições em frente à TV [20]. A pessoa, ao assistir TV, preocupa-se mais com a programação que com a qualidade dos alimentos consumidos. $\mathrm{O}$ acesso ilimitado aos recursos de tela deve ser evitado, e os pais e profissionais envolvidos com adolescentes devem desenvolver estratégias de análise do conteúdo apresentado na TV, computador e outros recursos eletrônicos, uma vez que eles parecem contribuir para a adoção de comportamentos de risco à saúde na juventude $[18,19]$.

Este estudo apresentou algumas limitações. Primeiro, a quantidade calórica e outros componentes alimentares (principalmente de alimentação saudável como frutas e verduras) não foram avaliados. A falta de uma análise multivariada e o fato da pesquisa ter sido realizada antes da era do smartfone, atualmente um dos maiores atrativos utilizados pelos adolescentes, podem ser consideradas outras limitações. Ainda, as medidas de hábitos alimentares, prática de atividade física e comportamentos sedentários foram autorreportados pelos adolescentes, e as estimativas podem ter sido sub ou superestimadas. Contudo, os instrumentos deste estudo são utilizados frequentemente com escolares [21]. Por fim, há possibilidade de causalidade reversa na relação entre tempo de tela e outros comportamentos (por exemplo, o hábito alimentar inadequado pode favorecer o tempo de tela, ao invés do contrário). Limitações estas que sugerem cautela na interpretação destes resultados.

Embora levando em conta estas limitações, pode-se concluir que fatores sociodemográficos (idade, sexo, situação de trabalho) e comportamentais (atividade física, hábitos alimentares), foram associados ao tempo de tela em adolescentes. Os resultados mostram ainda que os fatores associados diferiram de acordo com o tipo de aparelho mais utilizado (TV ou computador/ videogame). Estes aspectos devem ser considerados em programas de combate ao tempo sedentário no lazer em jovens. 


\section{REFERÊNCIAS}

1. World Health Organization WHO. Global status report on noncommunicable diseases. Geneva: 2010. [Cited 2014 December 10] Available from: http://www.who.int/nmh/publications/ncd_report_full_en.pdf

2. Maitland C, Stratton G, Foster S, Braham R, Rosenberg M. A place for play? The influence of the home physical environment on children's physical activity and sedentary behavior. Int J Behav Nutr Phys Act. 2013;10(99):1-21. http://dx.doi.org/10.1186/1479-5868-10-99

3. LeBlanc AG, Chaput JP, McFarlane A, Colley RC, Thivel D, Biddle SJ, Maddison R, Leatherdale ST, Tremblay MS.Active Video Games and Health Indicators in Children and Youth: A Systematic Review. Plos one. 2013; 8(6): 65351-71. http://dx.doi.org/10.1371/journal. pone.0065351

4. Trends in the prevalence of sexual behaviors. National Youth Risk Behavior Survey -YRBS; Atlanta: 2007. [Cited 2014 November 15] Available from: http://www.cdc.gov/healthyyouth/data/yrbs/results.htm

5. Autran RG, Rech CR, Mota J, Santos MP. Percepção de regras e de confiança em reduzir o tempo de tela em adolescentes. Rev Bras Ativ Fís Saúde. 2014;19(6):690-2. http://dx.doi.org/10.12820/rbafs.v.19n6p690

6. Dumith SC, Hallal PC, Menezes A, Araújo CL: Sedentary behavior in adolescents: the 11-year follow-up of the 1993 Pelotas (Brazil) birth cohort study. Cad Saude Publica. 2010;26(10):1928-36. http://dx.doi.org/10.1590/S0102-311X2010001000009

7. Cândido AP, Benedetto R, Castro AP, Carmo JS, Nicolato RL, Nascimento-Neto RM, Freitas RN, Freitas SN, Caiaffa WT, MachadoCoelho GL. Cardiovascular risk factors in children and adolescents living in an urban area of Southeast of Brazil: Ouro Preto Study. Eur J Pediatr. 2009;168:1373-82. http://dx.doi.org/10.1007/s00431-009-0940-1

8. Vasques DG, Lopes AS. Fatores associados à atividade física e aos comportamentos sedentários em adolescentes. Rev Bras Cineantropom Desempenho Hum. 2009;11:59-66.

9. Ouwens MA, Cebolla A, Strien TV. Eating style, television viewing and snacking in pre-adolescent children. Nutr Hosp. 2012; 27(4):1072-8. http://dx.doi.org/10.3305/nh.2012.27.4.5805

10. Campagnolo PDB, Vitolo MR, Gama CM. Fatores associados ao hábito de assistir TV em excesso entre adolescentes. Rev Bras Med Esporte. 2008;14(3):197-9. http://dx.doi.org/10.1590/S1517-86922008000300007

11. Fernandes RA, Christofaro DG, Casonatto J, Kawaguti SS, Ronque ER, Cardoso JR, Freitas Júnior IF, Oliveira AR. Cross-sectional association between healthy and unhealthy food habits and leisure physical activity in adolescents. J Pediatr. 2011;87(3):252-6. http:// dx.doi.org/10.2223/JPED.2093

12. Nunes MMA, Figueiroa JN, Alves JGB. Excesso de peso, atividade física e hábitos alimentares entre adolescentes de diferentes classes econômicas em Campina Grande (PB). Rev Assoc Med Bras. 1992;53(2):130-4.

13. Fiates GMR, Amboni RDMC, Teixeira E. Comportamento consumidor, hábitos alimentares e consumo de televisão por escolares de Florianópolis. Rev Nutr. 2008;21(1):105-14. http://dx.doi.org/10.1590/S1415-52732008000100011

14. Borghese MM, Tremblay MS, Leduc G, Boyer C, Bélanger P, LeBlanc AG, Francis C, Chaput JP. Independent and combined associations of total sedentary time and television viewing time with food intake patterns of 9- to 11-year-old Canadian children. Appl Physicol Nutri Metab. 2014;39(8):1-7. http://dx.doi.org/10.1139/apnm-2013-0551

15. Lissner L, Lanfer A, Gwozdz W, Olafsdottir S, Eiben G, Moreno LA, Santaliestra-Pasías AM, Kovács E, Barba G, Loit HM, Kourides Y, Pala V, Pohlabeln H, De Henauw S, Buchecker K, Ahrens W, Reisch L. Television habits in relation to overweight, diet and taste preferences in European children: the IDEFICS study. Eur J Epidemiol. 2012;27:705-15. http://dx.doi.org/10.1007/s10654-012-9718-2

16. BRASIL. Ministério do Planejamento Orçamento e Gestão. Ministério da Saúde. Instituto Brasileiro de Geografia e Estatística - IBGE. Pesquisa Nacional de saúde do escolar. Rio de Janeiro, 2009.

17. Centers for Disease Control and Prevention. Youth risk behavior surveillance - United States, 2007. MMWR. 2008; 57(4):1-130.

18. Friedrich RR, Polet JP, Schuch I, Wagner MB. Effect of intervention programs in schools to reduce screen time: a meta-analysis. J Pediatr. 2014;90(3):232-41. http://dx.doi.org/10.1016/j.jped.2014.01.003

19. Bucksch J, Inchley J, Hamrik Z, Finne E, Kolip P. Trends in television time, non-gaming PC use and moderate-to-vigorous physical activity among German adolescents, 2002-2010. BMC Public Health. 2014;14(351):1471-2458. http://dx.doi.org/10.1186/1471-2458-14-351

20. Totland TH, Bjelland M, Lien N, Bergh IH, Gebremariam MK, Grydeland M, Ommundsen Y, Andersen LF. Adolescents' prospective screen time by gender and parental education, the mediation of parental influences. Int J Behav Nutr Phys Act. 2013;10:89. http://dx.doi. org/10.1186/1479-5868-10-89

21. Nuutinen T, Ray C, Roos E. Do computer use, TV viewing, and the presence of the media in the bedroom predict school-aged children's sleep habits in a longitudinal study? BMC Public Health. 2013;13(1):684. http://dx.doi.org/10.1186/1471-2458-13-684 